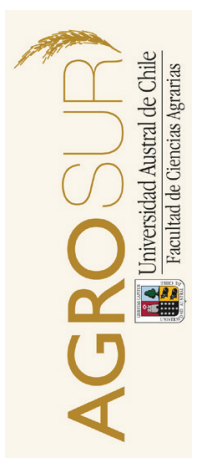

\title{
Acción biocida del jugo de fique (Furcraea gigantea Vent.) sobre Colletotrichum gloeosporioides aislado de tomate de árbol (Solanum betaceum Cav.)
}

\author{
Biocidal action of Fique (Furcraea gigantea Vent.) juice on Colletotrichum \\ gloeosporioides isolated from Tamarillo fruit (Solanum betaceum Cav.)
}

\author{
Santander, M. ${ }^{a *}$, Cerón, L. ${ }^{a}$, Hurtado, A $^{a}$. \\ ${ }^{a}$ Universidad de Nariño. Facultad de Ingeniería Agroindustrial. Grupo de Investigación Tecnologías \\ Emergentes en Agroindustria - TEA. Pasto, Colombia.
}

\begin{tabular}{l} 
A R T I C L E I N F O \\
\hline Article history: \\
Received: 02.09.14 \\
Accepted: 18.11 .14 \\
\hline Keywords: \\
Antifungal \\
Colletotrichum gloeosporioides \\
Anthracnose \\
Tamarillo fruit \\
\hline Original Research Article, \\
Food and Technology Science \\
\hline *Corresponding author: \\
Margareth Santander \\
E-mail address: \\
marguis santanderm@ \\
hotmail.com
\end{tabular}

\begin{abstract}
A B S T R A C T
The biocidal action of fique juice on the phytopathogen C. gloeosporioides, which causes anthracnose in tamarillo fruit was evaluated. Concentrations of 1,000, 10,000 and 100,000 $\mathrm{g} \mathrm{mL}^{-1}$ amended in the PDA culture medium were analyzed and tree levels of fermentation which were 0,4 and 8 days. Mycelial discs of $1.1 \mathrm{~cm}$ in diameter were placed in different experimental units and the in vitro growth area was measured after a period of 8 days using the ImageJ software. Only statistically significant differences $(\mathrm{P}<0.05)$ occurred between concentrations and between the interaction of two factors. The Maximum inhibition of the pathogen was obtained by using a concentration of $100,000 \mu \mathrm{g} \mathrm{mL}^{-1}$ and 8 days of fermentation.
\end{abstract}

\section{RESUMEN}

Se evaluó la acción biocida del jugo de fique frente al fitopatógeno C. gloeosporioides, causante de la antracnosis en tomate de árbol. Se analizaron concentraciones de 1.000, 10.000 y $100.000 \mu \mathrm{g} \mathrm{mL}^{-1}$ enmendadas en medio de cultivo PDA y tres niveles de fermentación que correspondieron a 0, 4 y 8 días. Discos de micelio de 1,1 cm de diámetro fueron colocados en las diferentes unidades experimentales y el área de crecimiento in vitro fue medido después de un periodo de 8 días utilizando el software ImageJ. Solo se presentaron diferencias estadísticamente significativas $(\mathrm{P}<0,05)$ entre las concentraciones estudiadas y entre la interacción de los dos factores. La máxima inhibición del patógeno se obtuvo al emplear una concentración de $100.000 \mu \mathrm{g}$ $\mathrm{mL}^{-1}$ y 8 días de fermentación.

Palabras clave: Antifúngico, Colletotrichum gloeosporioides, antracnosis, tomate de árbol.

\section{INTRODUCCIÓN}

Se precisa que dentro de los cultivos frutícolas del departamento de Nariño, el tomate de árbol (Solanum betaceum (av.) constituye una alternativa de gran importancia socioeconómica, pero en Colombia este se ve afectado por la antracnosis, la cual es una enfermedad de gran trascendencia debido a su amplia distribución y a la magnitud de las pérdidas post-cosecha que ocasiona, ya que si no se controla estas pueden llegar al 100\% (Saldarriaga et al., 2008).

La antracnosis es causada por el hongo C. gloeosporioides y ataca hojas, ramas jóvenes, inflorescencias y frutos, manifestándose con la presencia de manchas necróticas que afectan el valor comercial del producto (Lobo, 2004).

Dinham y Malik (2003), establecen que los plaguicidas sintéticos han generado beneficios en la producción agrícola, sin embargo el empleo inadecuado de los mismos, expresado en términos de tipo, toxicidad, número de aplicaciones y dosificación han producido contaminación sobre el suelo, agua, aire y productos agrícolas, por la acumulación de residuos potencialmente dañinos a la salud humana y de los animales. Por tanto, según Staufer et al. (2000), el interés por el uso de los extractos vegetales con este fin se ha incremen- 
tado considerablemente, con prometedores resultados de investigaciones in vitro e in vivo, con especies de plantas de diferentes ambientes ecológicos y abundantes en la naturaleza.

De acuerdo a los estudios realizados por Rojas (2008), se encontró que el jugo de fique (Furcraea gigantea Vent.) se puede utilizar como biofungicida debido a que posee metabolitos secundarios como saponinas y alcaloides, los cuales pueden ser los responsables de su actividad antifúngica. Además, su empleo en este tipo de productos contribuiría a disminuir el gran impacto ambiental que causa su vertimiento en ríos y quebradas después de su extracción.

El objetivo de la presente investigación fue estudiar la actividad biocida del jugo de fique sobre Colletotrichum gloeosporioides, causante de la antracnosis en el tomate de árbol.

\section{MATERIAL Y MÉTODO}

\section{Localización}

Esta investigación se llevó a cabo en el Laboratorio de Fitopatología de la Universidad de Nariño, ubicada al noroeste de la ciudad de San Juan de Pasto, departamento de Nariño, Colombia $\left(01^{\circ} 12^{\prime} 13^{\prime \prime}\right.$ N y $77^{\circ} 15^{\prime} 23^{\prime \prime}$ 0 ), a una altitud de 2.540 m.s.n.m y temperatura promedio de $18^{\circ} \mathrm{C}$.

\section{Aislamiento de Colletrotrichum gloeosporioides}

Se llevó a cabo el protocolo de Agrios (2005). El patógeno se aisló de una hoja infectada de árbol de tomate, seleccionando varios cortes pequeños de 5 a 10 $\mathrm{mm}^{2}$ a partir del borde de la lesión contagiada con el fin de obtener tejidos enfermos. Esos cortes se sumergieron en una solución de hipoclorito de sodio al 10\% y al cabo de 30 segundos se tomaron asépticamente y se secaron con trozos de papel estéril. Finalmente se inocularon sobre el medio nutritivo PDA acidificado con ácido sulfúrico al 98,5\%. El patógeno se purificó por transferencia continua a nuevas cajas de Petri hasta obtener una colonia pura de C. gloeosporioides.

\section{Adquisición y preparación del jugo de fique.}

Las pencas de fique variedad negra común fueron adquiridas del municipio de Nariño en el departamento de Nariño. Estas se sometieron a un proceso de lavado y posteriormente fueron desinfectadas con hipoclorito de sodio a una concentración de $100 \mu \mathrm{g} \mathrm{mL}^{-1}$.

La extracción del jugo de fique se realizó empleando un molino de rodillos y se filtró usando lienzos debido a la alta cantidad de residuos sólidos presentes. El jugo de fique utilizado en fresco se pasteurizó inmediatamente después de su extracción, mientras que el jugo fermentado durante 4 y 8 días se pasteurizó después del proceso de fermentación. El tratamiento térmico empleado fue $75^{\circ} \mathrm{C}$ durante $2 \mathrm{~min}$.

El jugo de fique se fermentó empleando la metodología expuesta por Álvarez et al. (2010), en la cual la fracción final filtrada de jugo fue fermentada de manera aeróbica a una temperatura constante de $33^{\circ} \mathrm{C}$ en una mufla. Este proceso se realizó en un periodo de 4 y 8 días.

\section{Sensibilidad del fitopatógeno Colletrotrichum gloeosporioides}

El comportamiento in vitro del patógeno se determinó con el área de crecimiento. Para esto, discos de micelio de 1,1 cm de diámetro de C. gloeosporioides obtenidos con sacabocado fueron establecidos en cajas Petri con medio PDA enmendado con las concentraciones de jugo de fique evaluadas: $1.000,10.000$ y 100.000 $\mu \mathrm{g} \mathrm{mL} \mathrm{m}^{-1}$. Los tratamientos se realizaron empleando volúmenes de 60, 600 y $6.000 \mu \mathrm{L}$ de jugo de fique, respectivamente, los cuales se introdujeron en matraces Erlenmeyers de $60 \mathrm{~mL}$ y se aforaron con medio de cultivo PDA. Para el caso del tratamiento control, los discos de micelio del fitopatógeno fueron inoculados en medio PDA sin adición de jugo de fique. Cada unidad experimental fue evaluada mediante el software Image después de un periodo de incubación de 8 días a temperatura promedio de $18^{\circ} \mathrm{C}$. El área de crecimiento $(A C)$ del fitopatógeno se determinó mediante la ecuación 1 :

$$
\mathrm{AC}=\mathrm{AMCT}-\mathrm{AS}
$$

Donde: $A M C T$ es el área media de la colonia creciendo en tratamiento y $A S$ es el área del sacabocado (Álvarez et al. 2010).

\section{Análisis estadístico}

Se empleó un diseño multifactorial categórico, con tres repeticiones por tratamiento, cada placa constituyó una unidad experimental. Se analizaron tres niveles de fermentación que correspondieron a 0 , 4 y 8 días y concentraciones de 1.000, 10.000 y $100.000 \mu \mathrm{g} \mathrm{mL}$. Los análisis de resultados se desarrollaron utilizando el software Statgraphics (C) Plus 5.0. Las diferencias estadísticas entre los resultados fueron calculadas mediante un análisis de varianza (ANOVA). Se empleó la Prueba de Rangos Múltiples (LSD de Fisher) para indicar las diferencias estadísticamente significativas entre las medias de los tratamientos evaluados.

\section{RESULTADOS Y DISCUSIÓN}

El análisis de varianza mostró que hubo diferencias estadísticamente significativas $(\mathrm{P}<0,05)$ entre las concentraciones de jugo de fique estudiadas y la interac- 
ción entre estas y los días de fermentación con respecto al área de crecimiento de C. gloeosporioides.

La Figura 1 presenta el comportamiento que exhibió el fitopatógeno entre los medios que fueron enmendados con las concentraciones analizadas. Se observó que el jugo de fique ejerce una acción biocida sobre C. gloeosporioides y que a mayores concentraciones su área de crecimiento disminuyó, encontrando que a $100.000 \mu \mathrm{g} \mathrm{mL}^{-1}$ se presentó el mínimo crecimiento del hongo. Por tanto, el crecimiento del micelio del fitopatógeno fue directamente influenciado por la concentración del extracto de fique.

En estudios realizados por Gómez (2002), se obtuvo que entre el 1,0 y 2,0\% hubo inhibición del micelio del patógeno y la afectación empieza a ser severa desde el $1 \%$ y se inhibe completamente a partir del $5 \%$.

También, en investigaciones in vitro desarrolladas por el MADR (2006), se determinó que el extracto de fique inhibe el desarrollo micelial de C. gloeosporioides cuando la concentración en el medio PDA es superior al 5\%.

Estos resultados presumiblemente se deben a la gran proporción de metabolitos secundarios presentes en el extracto de fique (Gómez y Vanegas, 2001; Preciado y Rangel, 2006), como alcaloides (Valencia et al., 2007), flavonoides, saponinas y triterpenos (Guenounea et al., 2001), los cuales están formados a través de las rutas de obtención de los metabolitos primarios y por procesos bioquímicos ayudados por enzimas provenientes de cadenas de oxidaciones y reducciones y han demostrado tener una fuerte actividad antibacterial y antifúngica (Sanabria, 1983; Verpoorte y Memelink, 2002). Así mismo, Fuertes et al. (1998), indican que la acción antifúngica de los alcaloides presentes en un extracto vegetal como el jugo de fique, podría deberse a la presencia de nitrógeno en su estructura como amina o amida interfiriendo en el desarrollo del patógeno a evaluar.
Al comparar los resultados obtenidos con los de otras investigaciones realizadas sobre maracuyá (Morillo, 1998), mango (Gutiérrez et al., 2003; Rondón et al., 2006) y papaya (González et al., 2007), las concentraciones de fungicidas sintéticos como Dithane, Imazalil, Prochloraz (Funcloraz), Benomil (Benlate), Mancozeb y sulfato de cobre (Phyton 27) empleadas para inhibir el crecimiento de C. gloeosporioides fueron menores al relacionarlas con las de jugo de fique utilizadas en esta investigación, ya que en el caso del tratamiento de antracnosis de maracuyá se presentó una inhibición total a partir de $100 \mu \mathrm{g} \mathrm{mL}^{-1}$ con Dithane y para el mango al usar Imazalil y Prochloraz se tuvo una concentración letal media a $39 \mu \mathrm{g} \mathrm{m} \mathrm{L}^{-1}$ y $21 \mu \mathrm{g} \mathrm{mL}^{-1}$, mientras que para inhibir al fitopatógeno con jugo de fique se requieren mayores concentraciones, obteniéndose una inhibición total a $100.000 \mu \mathrm{g} \mathrm{mL}^{-1}$.

Lo anterior, presumiblemente se debe a que el extracto de fique presenta $85 \%$ de humedad y tan solo el $8 \%$ del volumen total representa la parte orgánica y amorfa (MADR, 2006), en la cual se encuentra los principios activos (metabolitos secundarios) reportados como antifúngicos.

El análisis de varianza mostró que no hubo diferencias significativas $(P>0,05)$ entre los días de fermentación estudiados, lo cual posiblemente se explica debido a que en ensayos preliminares desarrollados por Benavides et al. (2010), en los cuales se realizó la detección cualitativa mediante reacciones colorimétricas y cromatografía de capa fina de metabolitos secundarios como flavonoides, cumarinas, saponinas, taninos, esteroides, glicósidos cardiotónicos, alcaloides y saponinas, en el jugo de fique fresco y fermentado de las variedades uña de águila y negra común procedentes de El Tambo y Guaitarilla (Nariño), se encontró una variabilidad de metabolitos secundarios como saponinas esteroidales y triterpénicas tanto en el jugo de fique fresco como en el jugo fermentado de las dos varieda-

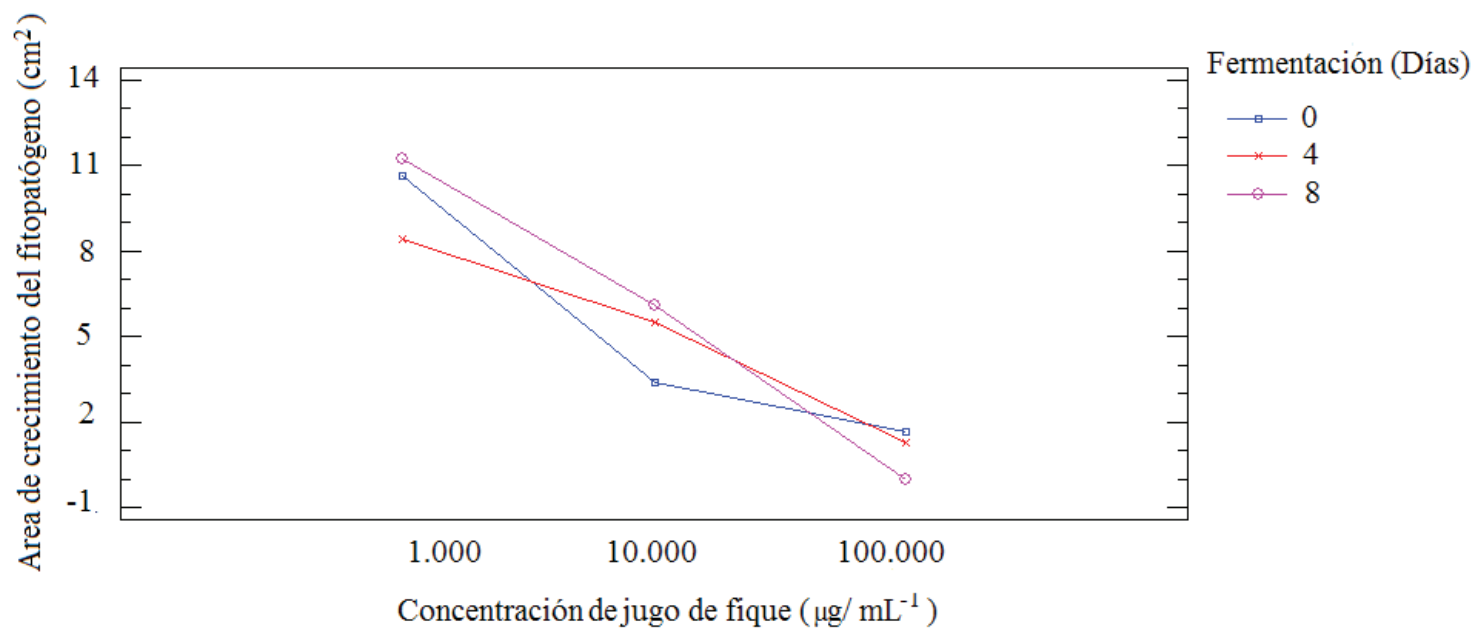

Figura 1. Comportamiento de C. gloeosporioides bajo diferentes concentraciones y días de fermentación de jugo de fique.

Figure 1. Behavior of $C$. gloeosporioides under different concentrations and fermentation days of fique juice. 
des. Por tal razón, al establecer que en las dos clases de jugo se encuentran los componentes que otorgan la acción biocida se infiere que no hubo diferencias entre los tratamientos, sin embargo, es necesario un análisis cuantitativo para conocer cómo influye la fermentación en la concentración de estos metabolitos.

Además según los efectos de interacción entre los factores evaluados en esta investigación (Figura 1), se obtuvo que en el día $8^{\text {vo }}$ de fermentación, a una concentración de $100.000 \mu \mathrm{g} \mathrm{mL}^{-1}$ se encontró la mayor inhibición de C. gloeosporioides. La Figura 2 muestra la comparación entre las diferentes concentraciones analizadas en este nivel de fermentación. La inhibición del crecimiento micelial al emplear saponinas se alude presumiblemente a la desactivación de la síntesis de varias enzimas a nivel de la célula y del metabolismo del fitopatógeno (Preciado y Rangel, 2006).

La investigación en este sentido debe continuar realizándose en campo y bajo condiciones de cultivo comerciales, con el fin de determinar la residualidad del producto en el tomate de árbol, su acción para el consumo humano y el medio ambiente, ya que su uso como biofungicida constituye una alternativa para reemplazar los insumos sintéticos, los cuales generan costos superiores al productor y su empleo se está restringiendo, ya que han producido respuestas ambientales y toxicológicas en la salud del consumidor.

\section{CONCLUSIONES}

Bajo condiciones in vitro, se demostró que el jugo de fique de la especie Furcraea gigantea Vent., ejerció un efecto biocida frente al fitopatógeno Colletotrichum gloeosporioides.

La inhibición en el crecimiento de Colletotrichum gloeosporioides aumentó a medida que se incrementó la concentración de jugo de fique, encontrando que el área mínima de crecimiento del fitopatógeno se presentó a una concentración de $100.000 \mu \mathrm{g} \mathrm{mL}^{-1}$ y empleando 8 días de fermentación del jugo de fique.

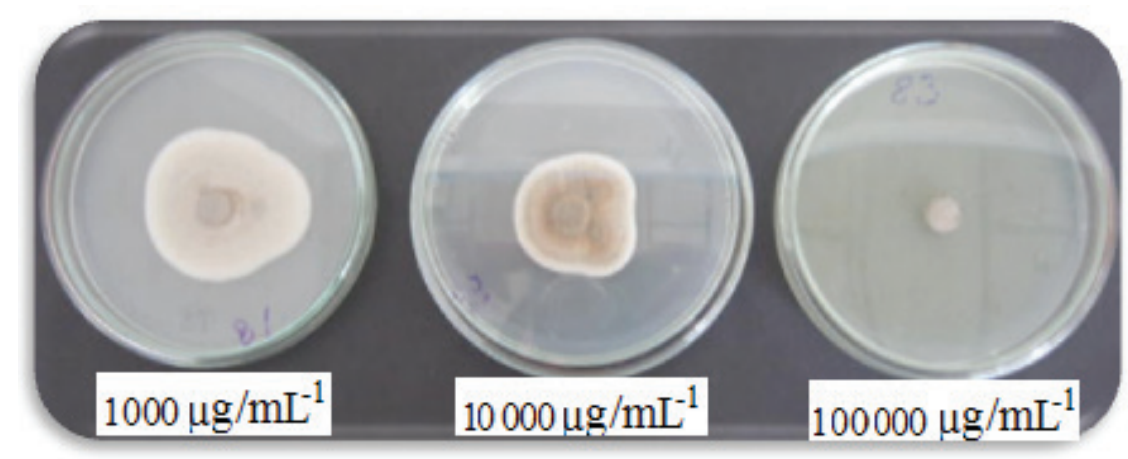

Figura 2. Sensibilidad del patógeno C. gloeosporioides bajo diferentes concentraciones de jugo de fique en el día $8^{\text {vo }}$ de fermentación.

Figure 2. Sensitivity of the C. gloeosporioides pathogen under different concentrations of fique juice in the day $8^{\text {th }}$ of fermentation.

\section{REFERENCIAS}

AGRIOS, G. 2005. Plant Pathology. Fifth Edition. Academic Press, New York.

ÁLVAREZ, D., DELGADO, D., SALAZAR, C., HURTADO, A., 2010. Sensibilidad in vitro de Phytophthora infestans (Mont) de Bary al extracto de fique (Furcraea gigantea Vent.) y fungicidas sistémicos. Tesis de Ingeniería Agronómica, Universidad de Nariño. 27 p.

BENAVIDES, O., HURTADO, A., ARANGO, O., BACCA, D., ROJAS, M. Estudio fitoquímico del jugo de fique de las variedades negra común y uña de águila de los municipios de El Tambo y Guaitarilla (Nariño-Colombia). XXIX Congreso Latinoamericano de Química, CLAQ Universidad de Nariño. Cartagena de Indias, Colombia. Del 27 de septiembre al 1 de octubre de 2010 .
DINHAM, B., MALIK, S. 2003. Pesticides and human rights. International Journal of Occupational and Environmental Health 9: 40-52.

FUERTES, C., ALCARRAZ, M., VIDALON, M. 1998. Flavonoides y alcaloides de Lupinus ballianus c.p. Smith con actividad antibacteriana y antifúngica. Lima, Perú. Instituto de Química Orgánica Aplicada a la Farmacia e Instituto de Microbiología, UNMSM. Facultad de Farmacia y Bioquímica. Ciencia e Investigación.

GÓMEZ, J. 2002. Evaluación del extracto de fique en el desarrollo in vitro de Colletotrichum gloeosporioides (Penz) Penz \& Sacc. y Sclerotinia sclerotiorum (Lib.) de bary .IV Seminario Nacional: Frutales de clima frío moderado. Medellín, Colombia. Del 20 al 22 Noviembre de 2002.

GÓMEZ, M., VANEGAS, E. 2001. Evaluación de la producción 
de esteroides a partir del jugo de fique con Cunninghamella spp. Tesis de grado, Universidad Pontificia Bolivariana de Medellín. 156 p.

GONZÁLEZ, N., SANDOVAL, C., GONZÁLEZ, E., MORALES, L. 2007. Sensibilidad in vitro de Colletotrichum gloeosporioides (Penz.) a fungicidas orgánicos derivados de extractos vegetales. IX congreso de ciencia de los alimentos y $\mathrm{V}$ foro de ciencia y tecnología de alimentos. Guanajuato, México. Del 31 de mayo a 1 de junio de 2007.

GUENOUNEA, D., GALILIA, S., PHILLIPS, D., VOLPIN, H., CHET, I. 2001. The defense response elicited by the pathogen Rhizoctonia solani is suppressed by colonization of the AM-fungus Glomus intraradices. Plant Science 160: 4449.

GUTIÉRREZ, J., GUTIÉRREZ, O., NIETO, D., TÉLIZ, D., ZAVALETA, E. 2003. Evaluación de Resistencia a Imazalil, Prochloraz y Azoxystrobin en Aislamientos de Colletotrichum gloeosporioides (Penz.) Penz. y Sacc. y Control de la Antracnosis del Mango (Mangifera indica L.) en Postcosecha. Revista Mexicana de Fitopatología 21: 379-383.

LOBO, M. 2004. Tomate de árbol (Cyphomandra betacea Sendt), frutal promisorio para la diversificación del agro andino. Corpoica. Medellín.

MINISTERIO DE AGRICULTURA Y DESARROLLO RURAL (MADR). 2006. Guía ambiental del subsector Fiquero. 2 ${ }^{\mathrm{a}}$ edición. Editorial Panamericana, Bogotá.

MORILLO, J. 1998. Estudio epidemiológico y evaluación de fungicidas contra la antracnosis (Colletotrichum gloeosporioides) del maracuyá para agricultura orgánica en Olancho, Honduras. Tesis de pregrado, Universidad de Zamorano. 48 p.
PRECIADO, D., RANGEL, E., 2006. Extracción de un biofungicida a partir del jugo de fique (Furcraea spp). Tesis de pregrado. Universidad Pontificia Bolivariana. Medellín. $78 \mathrm{p}$.

ROJAS, M. 2008. Caracterización fisicoquímica del jugo de fique (Furcraea spp.), elaboración y evaluación de un biofungicida útil en el control agroecológico de la gota (Phytopthora infestans) en la papa. Tesis de pregrado, Universidad de Nariño. 120 p.

RONDÓN, O., SANABRÍA, N., RONDON, A. 2006. Respuesta in vitro a la acción de fungicidas para el control de antracnosis, Colletotrichum gloeosporioides Penz, en frutos de mango. Agronomía Tropical 56: 219-235.

SALDARRIAGA, A., ZAPATA, J., ARANGO, I. 2008. Caracterización del agente causante de la antracnosis en tomate de árbol, manzano y mora. Academia Colombiana de Ciencias Exactas, Físicas y Naturales 32(123): 145-156.

SANABRIA, A. 1983. Análisis fitoquímico preliminar: Metodología y su aplicación en la evaluación de 40 plantas de familia Compositae. Universidad Nacional de Colombia, Bogotá.

STAUFER, A., ORREGO, A., AQUINO, A., 2000. Selección de extractos vegetales con efecto fungicida $\mathrm{y} / \mathrm{o}$ bactericida. Revista de Ciencia y Tecnología 1: 29-33.

VALENCIA, O., GUTIERREZ, J., SILVA, D., GÓMEZ, M., ISAZA, J. 2007. Actividad insecticida de extractos de Bocconia frutescens L. sobre Hypothenemus hampei F. Scientia et Technica 33: 23-27.

VERPOORTE, R., MEMELINK, J., 2002. Biotechnology for the production of plant secondary metabolites. Phytochemistry 1: 13-25. 
